Tohoku Math. J.

68 (2016), 439-456

\title{
THE LÊ-GREUEL FORMULA FOR FUNCTIONS ON ANALYTIC SPACES
}

\author{
Roberto Callejas-Bedregal, Michelle F. Z. Morgado \\ Marcelo Saia ANd José Seade
}

(Received June 17, 2014)

\begin{abstract}
In this article we give an extension of the Lê-Greuel formula to the general setting of function germs $(f, g)$ defined on a complex analytic variety $X$ with arbitrary singular set, where $f=\left(f_{1}, \ldots, f_{k}\right):(X, \underline{0}) \rightarrow\left(\mathbb{C}^{k}, \underline{0}\right)$ is generically a submersion with respect to some Whitney stratification on $X$. We assume further that the dimension of the zero set $V(f)$ is larger than 0 , that $f$ has the Thom $a_{f}$-property with respect to this stratification, and $g:(X, \underline{0})$ $\rightarrow(\mathbb{C}, 0)$ has an isolated critical point in the stratified sense, both on $X$ and on $V(f)$.
\end{abstract}

Introduction. We know from Milnor's book [28] that if $f$ is a holomorphic map-germ in $\mathbb{C}^{n+1}$ defining an isolated hypersurface singularity at the origin, then its Milnor number $\mu(f)$ can be computed as:

$$
\mu(f)=\operatorname{dim}_{\mathbb{C}} \frac{\mathcal{O}_{n+1, \underline{0}}}{\left(\frac{\partial f}{\partial z_{1}}, \ldots, \frac{\partial f}{\partial z_{n+1}}\right)} .
$$

Soon after Milnor's book was published, H. Hamm proved in [18] that every isolated complete intersection singularity germ (an ICIS for short) also has a well-defined Milnor number, defined as the rank of the middle homology of the corresponding Milnor fibre. It was thus natural to search for an algebraic expression for this invariant in the case of ICIS germs.

In 1970, motivated by earlier conversations with G. Tyurina, Lê Dũng Tráng gave such a formula in a seminar in Göttingen: If $f_{1}, \ldots, f_{k}$ and $g$ are holomorphic map germs $\left(\mathbb{C}^{n+k}, \underline{0}\right)$ $\rightarrow(\mathbb{C}, 0)$ such that $f=\left(f_{1}, \ldots, f_{k}\right)$ and $(f, g)$ define isolated complete intersection germs, then their Milnor numbers are related by:

$$
\mu(f)+\mu(f, g)=\operatorname{dim}_{\mathbb{C}} \frac{\mathcal{O}_{n+k, \underline{0}}}{\left(f, \operatorname{Jac}_{k+1}(f, g)\right)},
$$

where $\operatorname{Jac}_{k+1}(f, g)$ denotes the ideal generated by the determinants of all $(k+1)$ minors of the corresponding Jacobian matrix.

This formula appeared first quoted in K. Saito's paper of the 1972 Cargèse meeting (see [29]), though the actual proof by Lê, which was of a topological nature, was published till 1974, in [21]. In the meantime, in 1973, G.-M. Greuel gave in [16] an algebraic proof of this same formula, and so it has become known as the Lê-Greuel formula for the Milnor number.

2010 Mathematics Subject Classification. Primary 32S55; Secondary 14B05, 58K05, 32S05, 57P05.

Key words and phrases. Milnor and Milnor-Lê fibrations, Milnor numbers, Lê-Greuel formula, indices of vector fields, Whitney stratifications.

Research partially supported by CAPES, CNPq and FAPESP from Brasil, and CONACYT from México. 
We remark also that B. Teissier made in [33] a deep study of the topology of hypersurface singularities by taking slices by general linear planes. This led to the celebrated Teissier sequence of numbers, which are the Milnor numbers of the corresponding complete intersection germs one gets by taking linear slices of various dimensions; and he proved for these, interesting results which are reminiscent of the Lê-Greuel formula.

The goal of this article is extending the Lê-Greuel formula to the general setting of functions defined on a complex analytic variety $X$ with arbitrary singular set. Of course we need to impose some conditions on these functions in order to have Milnor type, or rather Milnor-Lê type, fibrations. We ask $f=\left(f_{1}, \ldots, f_{k}\right):(X, \underline{0}) \rightarrow\left(\mathbb{C}^{k}, \underline{0}\right)$ to be generically a submersion with respect to some Whitney stratification on $X$, the dimension of its zero set $V(f)$ must be larger than 0 , and $f$ must have the Thom property with respect to this stratification. And we ask $g:(X, \underline{0}) \rightarrow(\mathbb{C}, 0)$ to have an isolated critical point in the stratified sense, both on $X$ and on $V(f)$.

Notice that in this general setting neither one has the concept of a Milnor number, nor the algebraic invariant on the right hand side of the Lê-Greuel formula is necessarily preserved as we move away from the special fiber over 0 . Hence a first step towards generalizing that theorem is expressing it in terms that make sense in the general setting we envisage here. This is done as follows.

When $X$ is $\mathbb{C}^{n+k}$ and $f$ defines an ICIS germ, we know from [18] that its Milnor fibre $F_{f}$ is a bouquet of $\mu$ spheres of middle dimension, where $\mu$ is the Milnor number, and similarly for $(f, g)$. Also, in this case, the term on the right can be thought of as being the number $c\left(\left.g\right|_{F_{f}}\right)$ of critical points of the restriction $\left.g\right|_{F_{f}}$ of $g$ to a Milnor fibre of $f$, counted with their local multiplicities.

Hence the Lê-Greuel formula can be expressed as:

$$
\chi\left(F_{f}\right)=\chi\left(F_{f, g}\right)+(-1)^{n} c\left(\left.g\right|_{F_{f}}\right),
$$

where the first two terms are Euler characteristics of the corresponding Milnor fibers. Furthermore, the number $(-1)^{n} c\left(\left.g\right|_{F_{f}}\right)$ actually equals the $\operatorname{GSV}$-index $\operatorname{Ind}_{\mathrm{GSV}}(g, \underline{0}, f)$ of the gradient vector field of the restriction of $g$ to the hypersurface $V(f)$ defined by $f$. This invariant, the GSV-index, is defined for all vector fields on isolated complete intersection germs and provides an extension for singular varieties of the classical Poincaré-Hopf local index for vector fields on manifolds (see [3] for details). We thus get:

$$
\chi\left(F_{f}\right)=\chi\left(F_{f, g}\right)+\operatorname{Ind}_{\mathrm{GSV}}(g, \underline{0}, f) .
$$

That is the formulation we extend to the general setting described above.

Our work uses the theory of indices of vector fields on singular varieties, as we explain below. Let $X$ be a complex analytic variety of pure dimension $n+k$ in an open set containing the origin $\underline{0}$ in some complex space $\mathbb{C}^{m}$, and let $f:(X, \underline{0}) \rightarrow\left(\mathbb{C}^{k}, 0\right)$ be a holomorphic function which is generically a submersion with respect to some Whitney stratification $\left\{S_{\alpha}\right\}$ of $X$. We assume further that the zero set $V(f)$ has dimension greater than 1 and $f$ has the 
Thom $a_{f}$-property with respect to the above stratification. Denote by $F_{f}$ the corresponding Milnor fibre.

If $X$ is non-singular, then the GSV-index of $g$ on $V(f)$ is, by definition, the total PoincaréHopf index of an extension of $\nabla g$ to the Milnor fiber $F_{f}$. In the general setting the Milnor fibre $F_{f}$ is itself singular, hence such a definition makes no sense because the Poincare-Hopf index is defined only for vector fields on smooth manifolds. We use instead the Schwartz (also called radial) index of vector fields and define the GSV index of $g$ on $V(f)$ to be the total Schwartz index of an extension of $\nabla g$ to the Milnor fibre, see Section 2 for details. Then we prove (Theorem 3.1):

THEOREM (Generalized Lê-Greuel formula). Let $g:(X, \underline{0}) \rightarrow(\mathbb{C}, 0)$ have an isolated critical point at $\underline{0}$ in the stratified sense, both in $X$ and also in $V(f)$, and let $F_{f, g}$ be the Milnor fibre of the holomorphic map-germ $(f, g)$. Let us equip $F_{f}$ with the stratification obtained by intersecting this variety with the strata of $X$, and denote by $\operatorname{Ind}_{\mathrm{GSV}}(g, \underline{0}, f)$ the total GSV-index of $g$ on the Milnor fiber $F_{f}$. Then one has:

$$
\chi\left(F_{f}\right)=\chi\left(F_{f, g}\right)+\operatorname{Ind}_{\mathrm{GSV}}(g, \underline{0}, f) .
$$

In Section 1 of this article we set down the foundations of what we need in the sequel concerning Milnor type fibrations. And in Section 2 we develop the machinery we need about indices of vector fields. In Sections 3 and 4 we prove Theorem 3.1.

We notice that J. Damon in [7, Section 9] also gives a Lê-Greuel type formula which applies to the singular Milnor fiber of an almost free divisor in the smooth Milnor fiber of a non-isolated complete intersection singularity. We notice too that in [8] the authors use stratified Morse theory to give a Lê-Greuel type formula in the same setting we envisage in this paper, and they use that formula to prove the analogous statement for the local Euler obstruction of functions on singular varieties. This is an invariant introduced in [2], which provides an extension of the notion of the Milnor number to the case of functions on singular varieties. In Section 5 we discuss relations of our work with those articles, as well as with the Milnor number defined by J. W. Bruce and R. M. Roberts in [4].

We also remark that in [6], the authors adapted the techniques of this article to study the topology of real analytic map-germs, and they prove the analogous Lê-Greuel formula in that setting. And in the recent article [27, Theorem A5], Nuño-Ballesteros, Oréfice and Tomazella considered the case where $(X, \underline{0})$ is an Isolated Determinantal Singularity. They proved in that setting a formula similar to our Theorem 3.1.

In Section 6 we consider the invariant appearing in the right hand side of Theorem 3.1 and look at it from an algebraic point of view. This explains how Theorem 3.1 leads to the classical Lê-Greuel formula. In that setting one has the well-known equality:

$$
\operatorname{dim}_{\mathbb{C}} \frac{\mathcal{O}_{n+k, \underline{0}}}{\left(f, \operatorname{Jac}_{k+1}(f, g)\right)}=c\left(\left.g\right|_{F_{f}}\right),
$$

where the term on the right denotes the number of critical points of a Morsification of $g$ on $F_{f}$, which essentially coincides in this case with the corresponding GSV-index. Our formula 
in this article expresses the difference $\chi\left(F_{f}\right)-\chi\left(F_{f, g}\right)$ in terms of the corresponding GSVindex; and we know from [8] that this invariant is determined by the number of critical points in the various strata of the Milnor fibre $F_{f}$ of a Morsification of $g$, together with the Normal Morse data of the strata.

Of course it would be interesting to find an algebraic interpretation of this invariant, the corresponding GSV-index, in the general formula of Theorem 3.1. Alias, we do not know how to do that, not even in the apparently simpler case when the ambient space $X$ is $\mathbb{C}^{m}$. As we notice in Section 6, in that setting one has that generically, the critical points of $g$ on $F_{f}$ are isolated, and in that case the invariant in Theorem 3.1 can be expressed algebraically as,

$$
\operatorname{dim}_{\mathbb{C}} \frac{\mathcal{O}_{m, \underline{0}}}{\left(f-t, \operatorname{Jac}_{k+1}(f, g)\right)},
$$

where $f=t$ defines the Milnor fibre $F_{f}$. It is then natural to "specialize" this invariant to the fiber over 0 and this should yield to an expression for $c\left(\left.g\right|_{F_{f}}\right)$ in terms of the local algebra of $f$ and $g$ at $\underline{0}$. This works fine in the classical setting of the Lê-Greuel formula. The problem in general is that even if $g$ has finitely many critical points on $F_{f}$, if the germ of $V(f)$ at $\underline{0}$ has non-isolated singularities, then one has

$$
\operatorname{dim}_{\mathbb{C}} \frac{\mathcal{O}_{n+k, \underline{0}}}{\left(f, \operatorname{Jac}_{k+1}(f, g)\right)}=\infty,
$$

and we do not know if one can compute this invariant solely in terms of the local algebras of $f, g$ at $\underline{0}$.

The authors are grateful to professors Lê Dũng Tráng, Bernard Teissier and Mutsuo Oka, for very helpful conversations. They are also grateful to the Centro de Ciências Exatas e da Natureza, Universidade Federal da Paraíba, Brazil, to Instituto de Ciências Matemáticas e de Computação, Universidade de São Paulo, Brazil, to Instituto de Biociências, Letras e Ciências Exatas, Universidade Estadual Paulista, Brazil, and to Instituto de Matemáticas at Cuernavaca, Universidad Nacional Autónoma México, for their support and warm hospitality while working on this article.

1. Milnor-Lê fibrations on singular varieties. Throughout this article, $X$ will denote a complex analytic singular variety of dimension $n+k$ in an open set containing the origin $\underline{0}$ in some complex space $\mathbb{C}^{m}$, and $f:(X, \underline{0}) \rightarrow\left(\mathbb{C}^{k}, \underline{0}\right)$ is a holomorphic function which is generically a submersion with respect to some complex analytic Whitney stratification $\left\{S_{\alpha}\right\}$ of $X$. We assume further that the zero set $V:=V(f)$ has dimension more than 0 and $f$ has the Thom $a_{f}$-property with respect to the above stratification.

We recall (see for instance [15]) that a point $x \in X$ is a critical point of $f$, in the stratified sense, if the restriction of $f$ to the corresponding stratum has a critical point at $x$. These are the points such that $\operatorname{Jac}_{k}(f)(x)=0$, where $\operatorname{Jac}_{k}(f)$ denotes the ideal generated by the determinants of all the $k$ minors of the Jacobian matrix of the restriction of $f$ to the corresponding stratum. 
We denote by $\operatorname{Crit}(f)$ the critical locus of $f$, which is the union of all its critical points. The points in its complement are the regular points of $f$, and we denote by $\Delta_{f}:=f(\operatorname{Crit}(f))$ the discriminant of $f$. This is an analytic subset of $\mathbb{C}^{k}$.

Given $\varepsilon>\delta>0$ sufficiently small, we denote by $N(\varepsilon, \delta)$ the tube

$$
N(\varepsilon, \delta):=[\mathbb{B}(\varepsilon) \cap X] \cap f^{-1}\left(\mathbb{D}_{\delta}\right),
$$

where $\mathbb{B}(\varepsilon)$ is the ball of radius $\varepsilon$ around $\underline{0}$ in $\mathbb{C}^{m}$ and $\mathbb{D}_{\delta}$ is the ball in $\mathbb{C}^{k}$ of radius $\delta$ around 0 .

THEOREM 1.1. Let $g:(X, \underline{0}) \rightarrow(\mathbb{C}, 0)$ be holomorphic, with an isolated critical point at $\underline{0}$ in the stratified sense, both in $X$ and also in $V$. Then for every $\varepsilon>\varepsilon^{\prime}>0$ sufficiently small, there exists $\delta>0$ small enough with respect to $\varepsilon^{\prime}$ such that:

(1) One has a locally trivial fibration:

$$
f: N(\varepsilon, \delta) \backslash f^{-1}\left(\Delta_{f}\right) \longrightarrow \mathbb{D}_{\delta} \backslash \Delta_{f} .
$$

(2) Each fiber $F_{t}=f^{-1}(t) \cap \mathbb{B}_{\varepsilon}, t \in \mathbb{D}_{\delta} \backslash \Delta_{f}$ inherits a Whitney stratification from that in $X$, by intersecting $F_{t}$ with the strata of $X$.

(3) The critical points of $g$ in each fiber $F_{t}$ are all contained in the interior of the ball $\mathbb{B}_{\varepsilon^{\prime}}$.

PROOF. The proof of the first statement is standard, essentially imitating the arguments in [22]. In fact, choose $\varepsilon>0$ sufficiently small, so that every sphere in $\mathbb{C}^{m}$ centered at $\underline{0}$ and contained in the ball $\mathbb{B}_{\varepsilon}$ meets transversally every stratum in $V(f)$, i.e., $\mathbb{S}_{\varepsilon}$ is a Milnor sphere for $V$. The fact that such an $\varepsilon$ exists follows from Bertini-Sard's theorem in [36].

Since $f$ has the Thom property, this implies that every fiber $f^{-1}(t)$ sufficiently near $V$ also meets $\mathbb{S}_{\varepsilon}$ transversally. So the fact that one has the fibration in statement (i) is just a relative version of the classical Ehresman's fibration lemma. In fact, by [34] every Whitney stratification on a complex analytic space is Whitney strong, so in a neighbourhood of each fiber $F_{t}$ one can use Proposition 4.6 in [36] to lift vector fields in $\mathbb{C}^{k}$ to stratified, rugose vector fields on $X$, thus getting the topological product structure about each fiber.

The second statement in this theorem follows easily from [36] because $f$ is a submersion at each point in $F_{t}$.

Now let $g:(X, \underline{0}) \rightarrow(\mathbb{C}, 0)$ be a holomorphic germ with an isolated critical point at $\underline{0}$ in the stratified sense (see [15]). We assume further that $g$ also has an isolated critical point at $V$. In other words, we can assume $g$ is the restriction to $X$ of a holomorphic function $\tilde{g}$ in a neighbourhood $U$ of $\underline{0}$ in $\mathbb{C}^{m}$, whose gradient $\nabla \tilde{g}$ vanishes only at $\underline{0}$, and such that for each $z \in X$, the projection of $\nabla \tilde{g}(x)$ to the corresponding stratum is non-zero, unless $z=\underline{0}$.

Since $f$ has the Thom property with respect to this stratification, one has that for every $z \in V \backslash\{\underline{0}\}$, there is a neighbourhood $N_{z}$ such for each point $w$ in it, the projection of $\nabla \tilde{g}(w)$ to the stratum of the fiber $f^{-1}(f(w))$ that contains $w$ is non-zero. This implies the third statement in the theorem above. 
We remark that it is essential in the theorem above to demand that $f$ has the Thom property, for otherwise there may not be a Milnor fibration as shown by the famous example of Lê (see for instance [31, p. 23]; see also [12]).

From now on, given $X$ and $f$ as above, we call the fibration in Theorem 1.1 the MilnorLê fibration of $f$, and denote its fiber by $F_{f}$.

In the sequel we will consider too a holomorphic map-germ $g:(X, \underline{0}) \rightarrow(\mathbb{C}, 0)$ with an isolated critical point at $\underline{0}$, both on $X$ and also on $V(f)$ (for the given Whitney stratification). This implies that away from $\underline{0}$ the kernel of $d g$ is transversal to the corresponding stratum. It is thus an exercise to show that this implies that the map-germ $(f, g)$ also satisfies the Thom $a_{f}$ condition with respect to the given stratification. Thence one also has a Milnor-Lê fibration corresponding to the map-germ $(f, g)$. We denote the corresponding fiber by $F_{f, g}$.

2. Indices of vector fields on singular varieties. Let $X, f$ and $g$ be as before.

Definition 2.1. Let $\mathbb{B}_{r}$ be a closed ball in $\mathbb{C}^{m}$ such that each stratum in $X$ meets transversally the boundary sphere $\mathbb{S}_{r}=\partial \mathbb{B}_{r}$. Then we say that the intersection $X_{r}:=X \cap \mathbb{B}_{r}$ is a singular variety with boundary $\partial X_{r}:=X \cap \mathbb{S}_{r}$.

In this case the interior of $X \cap \mathbb{B}_{r}$ is a complex analytic singular variety in the usual sense, and the boundary is itself a real analytic (possibly singular) variety, which inherits a Whitney stratification from that in $X$.

Recall that a stratified vector field on $X$ means a section $v$ of the bundle $\left.T M\right|_{X}$, where $M$ is a complex manifold with a Whitney stratification adapted to $X$, such that for each $x \in X$ the vector $v(x)$ is tangent to the corresponding stratum. We further assume that all vector fields in this article are continuous.

We now recall the concept of the Schwartz index of a vector field. This index was introduced by H. King and D. Trotmann [19] under a different name. This was also studied by W. Ebeling and S. Gusein-Zade in several articles (e.g. [9]) where they call it the radial index. The presentation we make here basically comes from [3] where this is called the Schwartz index. Much of the presentation below is inspired also by the work of W. Ebeling and S. Gusein-Zade about the radial index for vector fields and for 1-forms (see for instance [10]).

Let $v$ be a stratified vector field on $X_{r}$ with isolated singularities. We want to define the Schwartz index of $v$ at these points. Since the question is local, we focus on a neighborhood of a singular point $x_{o}$.

Let us denote by $V_{\alpha}$ the stratum containing $x_{o}$. If the vector field $v$ is transversal to every small sphere in $\mathbb{C}^{m}$ centered at $x_{o}$, then we say that $v$ is radial. We denote such a vector field by $v_{\text {rad. }}$ In this case we define its Schwartz index to be 1 .

Now let $v$ be any stratified vector field on $X_{r}$ with an isolated singularity at a point $x_{o}$. We consider two balls $\mathbb{B}_{\varepsilon}, \mathbb{B}_{\varepsilon^{\prime}}$ centered at $x_{o}$, with $\varepsilon>\varepsilon^{\prime}>0$ without other singularity of $v$ and small enough so that their boundaries are transverse to all strata. Inside the smaller $\mathbb{B}_{\varepsilon^{\prime}}$ we consider a stratified radial vector field $v_{\text {rad }}$ with center $x_{o}$ and pointing outwards the ball. On the boundary $\partial \mathbb{B}_{\varepsilon}$ of the larger one, we consider the vector field $v$. 
Let us consider the cylinder $K_{\varepsilon, \varepsilon^{\prime}}=\left(\mathbb{B}_{\varepsilon} \backslash \operatorname{Int} \mathbb{B}_{\mathcal{\varepsilon}^{\prime}}\right) \cap X_{r}$. On the parts $\mathbb{S}_{\mathcal{\varepsilon}^{\prime}}=\partial \mathbb{B}_{\varepsilon^{\prime}}$ and $\mathbb{S}_{\varepsilon}=\partial \mathbb{B}_{\varepsilon}$ of the boundary of $K_{\varepsilon, \varepsilon^{\prime}}$ one has a vector field $w$ defined by $v_{\text {rad }}$ and $v$ respectively. One can always extend $w$ in $K_{\varepsilon, \varepsilon^{\prime}}$ by the classical radial extension process of M. H. Schwartz [30] (see for instance [3, Chapter 2, Section 3]). One has that at each singular point of the extension, the index in the stratum coincides with the index in the ambient space:

$$
\operatorname{Ind}_{\mathrm{PH}}\left(w, p_{j} ; V_{\beta}\right)=\operatorname{Ind}_{\mathrm{PH}}\left(w, p_{j} ; \mathbb{C}^{m}\right),
$$

where $V_{\beta}$ is the stratum containing $p_{j}$.

DEFINITION 2.2. The difference of $v$ and $v_{\text {rad }}$ is defined as:

$$
d\left(v, v_{\mathrm{rad}}\right)=\sum_{\beta} \sum_{j} \operatorname{Ind}_{\mathrm{PH}}\left(w, p_{j} ; V_{\beta}\right),
$$

where the sum on the right runs over the Poincare Hopf indices at the singular points of the restriction of $w$ to each stratum $V_{\beta}$ in $K_{\varepsilon, \varepsilon^{\prime}}$.

One has ([3, p. 38]) that this integer does not depend on the choice of $w$ (cf. [10, p. 441]) provided this extension is done by radial extension. Notice also that $d\left(v_{\mathrm{rad}}, v_{\mathrm{rad}}\right)=0$ and we can write

$$
\operatorname{ind}_{\operatorname{Sch}}\left(v, x_{o} ; X\right)=1+d\left(v, v_{\mathrm{rad}}\right) .
$$

Then we have a definition for the Schwartz (or radial) index of a vector field $v$ in a natural way:

DEFINITION 2.3. Let $X$ be a complex analytic singular variety, equipped with a Whitney stratification, and let $v$ be a continuous, stratified vector field on $X$ with isolated singularities $x_{1}, \ldots, x_{s}$. Then:

i) The Schwartz index of $v$ at each $x_{i} \in X$ is defined as:

$$
\operatorname{ind}_{\operatorname{Sch}}\left(v, x_{i} ; X\right)=1+d\left(v, v_{\mathrm{rad}}\right) .
$$

ii) The total Schwartz index of $v$ on $X$ is the sum of its local indices at the singular points.

The proof of the following theorem is exactly as that of Theorem 2.1.1 in [3] and we leave the details to the reader.

THEOREM 2.4. Let $X_{r}$ be a compact, complex analytic variety with boundary in $\mathbb{C}^{m}$ (or more generally in some complex manifold $M$ ), which is equipped with a Whitney stratifcation adapted to $X$. Let $v$ be a continuous, stratified vector field defined on a neighborhood of the boundary $\partial X_{r}$ in $\mathbb{C}^{m}$, with no singularities. Then:

1. It can also be extended to a stratified vector field on $X_{r}$ with isolated singularities $x_{1}, \ldots, x_{s}$, and its total Schwartz index in $X_{r}$ is independent of the choice of the extension.

2. If $v$ is transversal to the boundary $\partial X_{r}$ everywhere, then:

$$
\chi\left(X_{r}\right)=\sum_{i=1}^{s} \operatorname{Ind}_{\operatorname{Sch}}\left(v, x_{i} ; X_{r}\right),
$$


where $\chi\left(X_{r}\right)$ is the Euler-Poincaré characteristic.

We now consider another index of vector fields, the GSV-index. Classically (see [14]) one considers a hypersurface (or a complete intersection) germ $(V, \underline{0})$ defined by a holomorphic function $f:\left(\mathbb{C}^{n+1}, \underline{0}\right) \rightarrow(\mathbb{C}, 0)$ with an isolated critical point at $\underline{0}$. Then, given a vector field $v$ on $V$ with isolated singularity at $\underline{0}$, one can always extend it to a vector field $w$ on the Milnor fiber $F=F_{f}$ of $f$, with no singularities near the boundary. The total number of these singularities in $F$, counted with their local Poincaré-Hopf indices, is independent of the extension, and this number is, by definition, the GSV-index of $v$ at $\underline{0}$. That is:

$$
\operatorname{Ind}_{\mathrm{GSV}}(v, \underline{0} ; V):=\operatorname{Ind}_{\mathrm{PH}}(w ; F),
$$

where the term on the right is the total index.

This definition was extended in [3, Chapter 3] to the more general setting where $V$ can have non-isolated singularities, but the ambient space still is non-singular. Following that line of ideas, one may further extend this concept as follows. We let $X, f$ and $g$ be as in Section 1 above. That is, $(X, \underline{0})$ is a complex analytic variety of dimension $n+k$ in $\mathbb{C}^{m}$ with a singular point at the origin $\underline{0} ; f:(X, \underline{0}) \rightarrow\left(\mathbb{C}^{k}, \underline{0}\right)$ is a holomorphic function which is generically a submersion with respect to some Whitney stratification $\left\{S_{\alpha}\right\}$ of $X$, and such that its zero set $V:=V(f)$ has dimension more than 0 . We assume that $f$ has the Thom $a_{f}$-property with respect to this stratification. Finally, $g:(X, \underline{0}) \rightarrow(\mathbb{C}, 0)$ is a holomorphic map with an isolated critical point at $\underline{0}$ in the stratified sense, both in $X$ and also in $V$.

Let us describe now the construction in [2] of a stratified vector field on $V$, denoted by $\bar{\nabla}_{V}(g)$. Let us denote by $\bar{\nabla} \tilde{g}(x)$ the gradient vector field of $\tilde{g}: U \rightarrow \mathbb{C}$ at a point $x$ in the neighborhood $U$ of $\underline{0}$ in $\mathbb{C}^{m}$ with $\left.\tilde{g}\right|_{X}=g$, defined by

$$
\bar{\nabla} \tilde{g}(x):=\left(\frac{\overline{\partial \tilde{g}}}{\partial x_{1}}, \ldots, \frac{\overline{\partial \tilde{g}}}{\partial x_{m}}\right),
$$

where the bar denotes complex conjugation. Consider $V$ with Whitney stratification obtained by intersecting this variety with the strata of $X$, denoted by $\left\{V_{\alpha}\right\}$, and denote by $V_{\alpha}(x)$ the stratum containing $x$. Since $g:(X, \underline{0}) \rightarrow(\mathbb{C}, 0)$ has an isolated singularity at $\underline{0}$, the projection of $\bar{\nabla} \tilde{g}(x)$ on $T_{x} V_{\alpha}(x)$, denoted by $\hat{\zeta}_{\alpha}(x)$, is not zero. Gluing together the vector fields $\hat{\zeta}_{\alpha}$, obtain a stratified vector field on $V$, denoted by $\bar{\nabla}_{V}(g)$. This vector field is homotopic to $\left.\bar{\nabla} \tilde{g}\right|_{V}$ (the justification for this can be seen in [2, Section 2]). Now consider $g$ restricted to Milnor fiber $F_{f}$ with the Whitney stratification obtained by intersecting this variety with the strata of $X$. Just in the same way, obtain a stratified vector field on $F_{f}$ using, for each $x \in F_{f}$, the projection of $\bar{\nabla} \tilde{g}(x)$ on the tangent space to each stratum of $F_{f}$ containing $x$, denoted by $\bar{\nabla}_{F_{f}}(g)$. This vector field is homotopic to $\left.\bar{\nabla} \tilde{g}\right|_{F_{f}}$.

Now we define (compare with Definition 3.4.1 of [3]):

DEFINITION 2.5. The $G S V$-index of $g$ on $V$ relative to the function $f$ is, by definition, the total Schwartz index of the conjugate gradient vector field $\bar{\nabla}_{F_{f}}(g)$ on the Milnor fiber $F_{f}$ :

$$
\operatorname{Ind}_{\mathrm{GSV}}(g, \underline{0} ; f):=\operatorname{Ind}_{\mathrm{Sch}}\left(\bar{\nabla}_{F_{f}}(g) ; F_{f}\right) .
$$


It is clear that the same definition adapts easily to define the corresponding index for 1 -forms, in the vein of $[10,11]$. In fact in that case the definition is actually simpler because one does not need to extend vector fields from the singular variety to the Milnor fibre, but we only need to consider the restriction of the 1 -form $d g$ to the boundary of $F_{f}$.

3. The Lê-Greuel formula. We consider again $X, f$ and $g$ as before. So that $f$ : $(X, \underline{0}) \rightarrow\left(\mathbb{C}^{k}, \underline{0}\right)$ defines $V=V(f) ; X$ is equipped with a Whitney stratification $\left\{S_{\alpha}\right\}$ with respect to which $f$ is generically a submersion and it has the Thom property, and $g$ has an isolated critical point in $X$ and in $V$, with respect to this stratification. As before, we assume $V(f)$ has dimension $\geq 2$.

We know from Section 1 that $f$ has a Milnor-Lê fibration; we denote by $F_{f}$ the corresponding fiber. Notice that the fact that $g$ has an isolated critical point in $V$ implies that $(f, g)$ is also generically a submersion with the Thom property with respect to the stratification $\left\{S_{\alpha}\right\}$, and one has that the variety $V(f, g)$ has dimension at least 1 . So, by the same arguments as in Section 1, the map $(f, g)$ also has a Milnor-Lê fibration. We denote by $F_{f, g}$ the corresponding Milnor fiber. We have:

THEOREM 3.1 (The Lê-Greuel formula). One has:

$$
\chi\left(F_{f}\right)=\chi\left(F_{f, g}\right)+\operatorname{Ind}_{\mathrm{GSV}}(g, \underline{0} ; f) .
$$

In order to prove Theorem 3.1, the first step is choosing appropriate representatives of the Milnor fibers $F_{f}$ and $F_{f, g}$. For this we consider first a Milnor sphere $\mathbb{S}_{\varepsilon}$ for $X$ and $V(f)$ at $\underline{0}$, and the Milnor-Lê fibration of $f$ given by Theorem 1.1.

Since $g$ has an isolated critical point in $V:=V(f)$, by [22], there exists $\delta^{\prime}>0$ such that if we let $\mathbb{D}_{\delta^{\prime}}^{2}$ be the disc in $\mathbb{C}$ of radius $\delta^{\prime}$ about 0 and set

$$
N_{V}\left(\varepsilon, \delta^{\prime}\right)=g^{-1}\left(\mathbb{D}_{\delta^{\prime}}^{2}\right) \cap V(f) \cap \mathbb{B}_{\varepsilon},
$$

then

$$
g: N_{V}\left(\varepsilon, \delta^{\prime}\right) \backslash V(g) \longrightarrow \mathbb{D}_{\delta^{\prime}}^{2} \backslash\{0\},
$$

is a locally trivial fiber bundle, where $V(g):=g^{-1}(0)$. Choose a typical fiber $g^{-1}\left(s_{o}\right) \cap V(f)$ of this fibration, so $s_{o} \neq 0$ with $\left|s_{o}\right| \leq \delta^{\prime}$.

Now consider $\varepsilon^{\prime}>0$ small enough, so that the ball $\mathbb{B}_{\mathcal{E}^{\prime}}$ in $\mathbb{C}^{m}$ does not meet the fiber $g^{-1}\left(s_{o}\right)$. We use Theorem 1.1 again, to choose a Milnor fiber $F_{f}:=f^{-1}\left(t_{o}\right) \cap \mathbb{B}_{\varepsilon}$ for $f$, such that the restriction of $g$ to $F_{f}$ has no critical points away from $\mathbb{B}_{\varepsilon^{\prime}}$. Then the hypersurface $g^{-1}\left(s_{o}\right)$ meets $F_{f}$ transversally and therefore the intersection $F_{f, g}:=F_{f} \cap g^{-1}\left(s_{o}\right)$ serves as a model for the Milnor fiber of the Milnor-Lê fibration of $(f, g)$.

From now on we set $F_{f, g}:=F_{f} \cap g^{-1}\left(s_{o}\right)$ with $F_{f}=f^{-1}\left(t_{o}\right) \cap \mathbb{B}_{\varepsilon}$, and we equip $F_{f}$ and $F_{f, g}$ with the Whitney stratifications obtained by intersecting these varieties with the strata of $X$. We have:

LEMMA 3.2 (Main Lemma). There exists a continuous, stratified vector field $v$ on $F_{f}$ with the following properties: 
1. Restricted to a neighbourhood of its boundary $\partial F_{f}$, it is transversal to the boundary, pointing outwards.

2. It is tangent to the hypersurface $F_{f, g}$.

3. Within the ball $\mathbb{B}_{\varepsilon^{\prime}}$, $v$ is the vector field $\bar{\nabla}_{V}(g)$.

4. Away from $\mathbb{B}_{\mathcal{E}^{\prime}}$, $v$ has only isolated singularities, all contained in $F_{f, g}$, and at each of these singular points, the vector field is transversally radial to the stratum containing the singular point of $v$ (that is, $v$ is transversal to the boundary of every tubular neighbourhood of the stratum).

Notice that property 4 implies that the Schwartz index of $v$ at each singularity in $F_{f, g}$ equals the local Poincaré-Hopf index at that point, of the restriction of $v$ to the stratum.

Theorem 3.1 follows easily from this Lemma. In fact, the first property implies that the total Schwartz index of $v$ in $F_{f}$ is $\chi\left(F_{f}\right)$, by Theorem 2.4. Similarly, properties 1 and 2, together with Theorem 2.4, imply that the total Schwartz index of $v$ in $F_{f, g}$ is $\chi\left(F_{f, g}\right)$. Thus, again by Theorem 2.4, together with property 4 , we get that the difference $\chi\left(F_{f}\right)-\chi\left(F_{f, g}\right)$ equals the sum of the Schwartz indices of $v$ away from $F_{f, g}$, and this is the GSV-index of $\bar{\nabla}_{V}(g)$ by property 3 , since $v$ has no other singularities away from $F_{f, g}$ but those in $\mathbb{B}_{\varepsilon^{\prime}}$, where $v$ coincides with $\bar{\nabla}_{V}(g)$.

4. Proof of the main lemma. Lemma 3.2 and its proof are very much inspired by [1] and [2], where the authors prove similar statements to get Lefschetz type theorems for the local Euler obstruction. This lemma is in fact an immediate consequence of the three lemmas below.

Lemma 4.1. Let $\eta^{\prime}>0$ be small enough with respect to $\delta^{\prime}$, so that the disc $\mathbb{D}_{\eta^{\prime}}\left(s_{o}\right) \subset$ $\mathbb{C}$ centered at $s_{o}$, is contained in the interior of $\mathbb{D}_{\delta^{\prime}}$ and $g^{-1}\left(\mathbb{D}_{\eta^{\prime}}\left(s_{o}\right)\right)$ does not intersect $\mathbb{B}_{\varepsilon^{\prime}}$. Then, there exists a stratified vector field $w_{r}$ on $F_{f}$ satisfying:

1. Its restriction to $g^{-1}\left(\mathbb{D}_{\delta^{\prime}}\right) \cap \mathbb{S}_{\varepsilon}$ is tangent (stratified) to all the fibers $g^{-1}(s)$, and it is transversal to $\mathbb{S}_{\varepsilon}$, pointing outwards.

2. It is tangent to the fiber $g^{-1}\left(s_{o}\right)$, where it has only isolated singularities, and at each singularity, $w_{r}$ is transversally radial in $g^{-1}\left(s_{o}\right)$, to the stratum that contains that zero of $w_{r}$.

3. It is tangent to each fiber $g^{-1}(s)$, for $s \in \mathbb{D}_{\eta^{\prime}}\left(s_{o}\right)$.

PROOF. First we observe that we can use the classical method of Schwartz to construct a vector field $r$ on $g^{-1}\left(s_{o}\right)$ satisfying the item 2, as done in the proof of [1, Lemma 3.4], see also [2, Lemma 4.1]. This method consists of constructing the vector fields by induction on the dimensions of the strata. Therefore we obtain a vector field $r$ which is tangent to $g^{-1}\left(s_{o}\right)$, where it has only isolated singularities and at each singularity it is transversally radial in $g^{-1}\left(s_{o}\right)$, to the stratum that contains that singularity. As $g^{-1}\left(s_{o}\right)$ meets $f^{-1}\left(t_{o}\right) \cap \mathbb{B}_{\varepsilon}$ transversally, $w_{r}:=\left.r\right|_{f^{-1}\left(t_{o}\right) \cap \mathbb{B}_{\varepsilon}}$ satisfies the same property.

From the choice of the appropriate representatives of the Milnor fibers $F_{f}$ and $F_{f, g}$ we see that $g$ determines a locally trivial fibration of $g^{-1}\left(\mathbb{D}_{\delta^{\prime}}\right) \cap f^{-1}\left(t_{o}\right) \cap \mathbb{B}_{\varepsilon} \backslash g^{-1}(0)$ over $\mathbb{D}_{\delta^{\prime}} \backslash\{0\}$. 

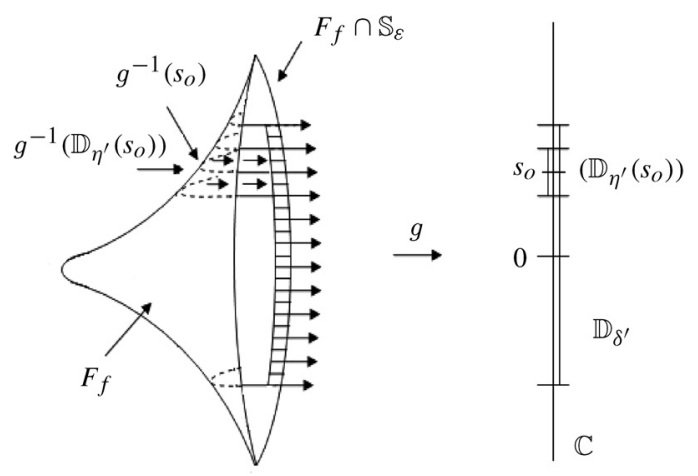

FIGURE 1. The vector field $w_{r}$.

This induces, in particular, a trivial fibration over $\mathbb{D}_{\eta^{\prime}}\left(s_{o}\right)$. Hence $w_{r}$ can be extended, as a product, to all fibers $g^{-1}(t) \cap f^{-1}\left(t_{o}\right)$ with $t \in \mathbb{D}_{\eta^{\prime}}\left(s_{o}\right)$, satisfying statement 3 .

To show the item 1 , we first choose $\varepsilon^{\prime \prime}$, with $\varepsilon^{\prime} \ll \varepsilon^{\prime \prime}<\varepsilon$ such that the restriction of $g$ to $g^{-1}\left(\mathbb{D}_{\delta^{\prime}}\right) \cap f^{-1}\left(t_{o}\right) \cap\left(\mathbb{B}_{\varepsilon} \backslash\right.$ Int $\left.\mathbb{B}_{\varepsilon^{\prime \prime}}\right)$ is a trivial fibration. Since $\mathbb{D}_{\delta^{\prime}}$ retracts to $\mathbb{D}_{\eta^{\prime}}\left(s_{o}\right)$, the vector field $w_{r}$ can be extended to $\mathbb{S}_{\varepsilon} \cap g^{-1}\left(\mathbb{D}_{\delta^{\prime}}\right) \cap f^{-1}\left(t_{o}\right)$ being tangent to the fibers of $g$ and transversal to the sphere $\mathbb{S}_{\varepsilon}$, pointing outwards. Using a suitable partition of unity, we can extend $w_{r}$ as zero in the complement of a neighborhood of $\left(g^{-1}\left(\mathbb{D}_{\delta^{\prime}}\right) \cap f^{-1}\left(t_{o}\right) \cap\left(\mathbb{B}_{\varepsilon} \backslash \operatorname{Int} \mathbb{B}_{\varepsilon^{\prime \prime}}\right)\right)$ $\cup\left(g^{-1}\left(\mathbb{D}_{\eta^{\prime}}\left(s_{o}\right)\right) \cap f^{-1}\left(t_{o}\right)\right)$.

LEMMA 4.2. There exists a stratified vector field $u$ defined on $F_{f} \cap g^{-1}\left(\mathbb{D}_{\delta^{\prime}}\right)$ minus the interior of the ball $\mathbb{B}_{\varepsilon^{\prime}}$, satisfying the following:

1. $u$ is tangent to $\mathbb{S}_{\varepsilon}$;

2. its zero set is $g^{-1}\left(s_{o}\right)$, and $u$ is transversally radial to $g^{-1}\left(s_{o}\right)$ in $F_{f}$;

3. $u$ is transversal to $F_{f} \cap g^{-1}\left(\partial \mathbb{D}_{\delta^{\prime}}\right)$, pointing outwards;

4. restricted to $F_{f} \cap \partial \mathbb{B}_{\varepsilon^{\prime \prime}}$ it coincides with the gradient vector field $\left.\bar{\nabla} g\right|_{F_{f}}$.

PROOF. Let $\left\{V_{\alpha}\right\}$ be $V_{\alpha}:=S_{\alpha} \cap F_{f}$ a Whitney stratification of $F_{f}$, where $\left\{S_{\alpha}\right\}$ is a Whitney stratification of $X$ as above. Since $g$ has isolated critical point in $X$ and $V(f)$ with respect to stratification $\left\{S_{\alpha}\right\}$ and $g^{-1}\left(s_{o}\right)$ meets $F_{f}:=f^{-1}\left(t_{o}\right) \cap \mathbb{B}_{\varepsilon}$ transversally, the restriction of $g$ to every stratum $V_{\alpha}$ (other than $\{\underline{0}\}$ itself) is regular. Therefore, the kernel of $d g$ has codimension 1 in $V_{\alpha}$.

The Hermitian metric on $\mathbb{C}^{m}$ induces a metric on $V_{\alpha}$ and defines a splitting of the tangent bundle $T V_{\alpha}$ as the sum of the tangent bundle to the fiber and the normal bundle. The derivative $d g$, restricted to the normal bundle, is an isomorphism. Hence, we can lift every vector field on $\mathbb{D}_{\delta^{\prime}}$ as a vector field tangent to $V_{\alpha}$ and orthogonal to the fibers $g^{-1}(t)$. Let us denote by $\xi$ the vector field on $\mathbb{D}_{\delta^{\prime}}$, radial from $s_{o}$, and by $u_{\alpha}$ the lifting of $\xi$ to $V_{\alpha}$.

Just as in the definition of the vector field $\left.\bar{\nabla} \tilde{g}\right|_{F_{f}}$, the Whitney conditions allow us to glue the different $u_{\alpha}$ in a stratified vector field $u$ defined on $g^{-1}\left(\mathbb{D}_{\delta^{\prime}}\right) \cap f^{-1}\left(t_{o}\right) \cap\left(\mathbb{B}_{\varepsilon} \backslash \operatorname{Int} \mathbb{B}_{\varepsilon^{\prime}}\right)=$ 

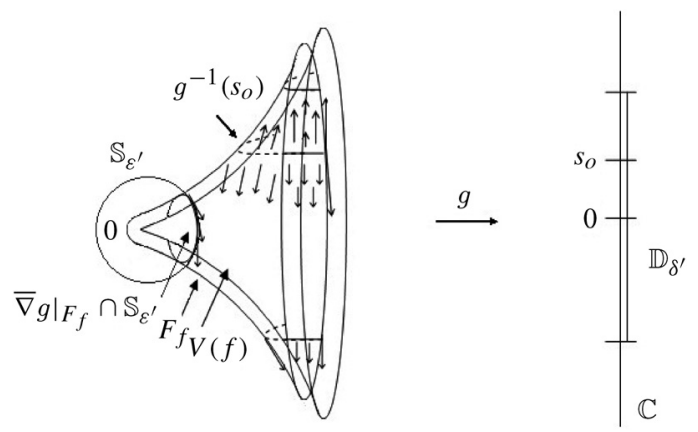

FIGURE 2. The vector field $u$.

$\left(F_{f} \cap g^{-1}\left(\mathbb{D}_{\delta^{\prime}}\right)\right) \backslash \operatorname{Int} \mathbb{B}_{\mathcal{E}^{\prime}}$ and satisfying the first three conditions of the lemma. The last statement follows as in the proof of Lemma 3.2 in [2, p. 69].

LEMMA 4.3. Let $w$ be a stratified vector field on $F_{f} \cap g^{-1}\left(\partial \mathbb{D}_{\delta^{\prime}}\right)$, which is transverse to both $F_{f} \cap \mathbb{S}_{\varepsilon}$ and $F_{f} \cap g^{-1}\left(\partial \mathbb{D}_{\delta^{\prime}}\right)$, pointing outwards. Then there exists an extension of $w$ to a stratified vector field on $F_{f} \backslash g^{-1}\left(\mathbb{D}_{\delta^{\prime}}\right)$, which is transverse to $\partial F_{f}=F_{f} \cap \mathbb{S}_{\varepsilon}$ and pointing outwards.

If in this statement we replace $F_{f}$ by $V(f)$, then the lemma is a special case of Theorem 2.3 in [1]. Lemma 4.3 then follows from this and the fact that $f$ has the Thom property.

\section{Remarks on the general setting.}

5.1. Damon's Lê-Greuel formula. J. Damon in [7, Section 9] gives a Lê-Greuel type formula which applies to the singular Milnor fiber of an almost free divisor in the smooth Milnor fiber of a non-isolated complete intersection singularity.

More precisely, consider almost free divisors $V_{i} \subset \mathbb{C}^{n}, 1 \leq i \leq k$ and define $V=$ $\bigcap_{i=1}^{k} V_{i}$ and $\tilde{V}=\bigcap_{i=1}^{k-1} V_{i}$, such that both $V$ and $\tilde{V}$ are almost free complete intersections. For sufficiently small $t$ and $s$ with $|t| \ll s$, Damon constructs in [7, p. 80] the smooth Milnor fiber $\tilde{V}_{t, s}$ for $\tilde{V}$, the singular Milnor fiber $V_{k, t}$ for $V_{k}$ and defines the relative Euler characteristic of the pair $(\tilde{V}, V)$ by

$$
\tilde{\chi}(\tilde{V}, V):=\chi\left(\tilde{V}_{t, s} \cap \mathbb{B}_{\varepsilon}, \tilde{V}_{t, s} \cap V_{k, t} \cap \mathbb{B}_{\varepsilon}\right) .
$$

Then by [7, Theorem 9.4] one has that $\tilde{\chi}(\tilde{V}, V)=(-1)^{n-k+1} \operatorname{dim}_{\mathbb{C}} D(\tilde{V}, V)$, where $D(\tilde{V}, V)$ is a determinantal module given as a quotient module of $\mathcal{O}_{\tilde{V}, \underline{0}}^{p}$ by a set of generators of $\operatorname{Derlog}\left(V_{i}^{\prime}\right)$ and $\left\{\frac{\partial \varphi}{\partial x_{1}}, \ldots, \frac{\partial \varphi}{\partial x_{n}}\right\}$. When $V$ is an ICIS one has a "module version" of the LêGreuel formula:

$$
\mu(\tilde{V})+\mu(V)=\operatorname{dim}_{\mathbb{C}} \frac{\mathcal{O}_{\tilde{V}, \underline{0}}^{p}}{\mathcal{O}_{\tilde{V}, \underline{0}}\left\{\frac{\partial \varphi}{\partial x_{1}}, \ldots, \frac{\partial \varphi}{\partial x_{n}}\right\}} .
$$


5.2. The Lê-Greuel formula of Dutertre-Grulha. In a recent paper, N. Dutertre and N. G. Grulha Jr. prove a Lê-Greuel formula which holds in the same general setting we envisage in this article, Theorem 4.5 in [8]. They express the difference $\chi\left(F_{f}\right)-\chi\left(F_{f, g}\right)$ appearing in Theorem 3.1 as a sum:

$$
\chi\left(F_{f}\right)-\chi\left(F_{f, g}\right)=\sum(-1)^{d_{\alpha}} \sum \mu_{i j}\left(1-\chi\left(\mathrm{lk}^{\mathbb{C}}\left(S_{\alpha}, X\right)\right),\right.
$$

where the first sum on the right runs over the strata $S_{\alpha}$ in the Milnor fibre of $f$, the number $d_{\alpha}$ being the dimension of the stratum; the $\mu_{i j}$ are certain multiplicities that can be described as the number of critical points, in each stratum of the Milnor fibre $F_{f}$, of a Morsification of $g$, and $\chi\left(\mathrm{k}^{\mathbb{C}}\left(S_{\alpha}, X\right)\right)$ is the Euler characteristic of the complex link of the corresponding stratum.

Using this formula together with our Theorem 3.1 one gets an interpretation of the invariant $\operatorname{Ind}_{\mathrm{GSV}}(g, \underline{0} ; f)$ as:

$$
\operatorname{Ind}_{\mathrm{GSV}}(g, \underline{0} ; f)=\sum(-1)^{d_{\alpha}} \sum \mu_{i j}\left(1-\chi\left(\mathrm{lk}^{\mathbb{C}}\left(S_{\alpha}, X\right)\right) .\right.
$$

In other words, when the fiber $F_{f}$ is smooth, then it is well known that the GSV-index of the gradient vector field of $g$ on the hypersurface defined by $f, \operatorname{Ind}_{\mathrm{GSV}}(g, \underline{0} ; f)$, is up to sign the number of critical points of a Morsification of $g$ on $F_{f}$. The above expression generalizes that interpretation of $\operatorname{Ind}_{\mathrm{GSV}}(g, \underline{0} ; f)$ to the case when the Milnor fibre $F_{f}$ is itself singular.

We notice too that in [24] the authors introduced certain polar invariants (or multiplicities) that detect the local Euler obstruction of a singular variety at each point. A similar study was done in [32] to express the local Euler obstruction of a function on a singular variety. In the setting we envisage here, we can also think of the $\operatorname{index}_{\operatorname{Ind}} \operatorname{GSV}_{(}(\underline{0} ; f)$ as a polar invariant (cf. [8, Section 5]).

5.3. Bruce-Roberts' Milnor number. In [4], J. W. Bruce and R. M. Roberts introduced a Milnor number for function germs on singular varieties. To recall this, let $W$ be a sufficiently small representative of a reduced germ $(W, \underline{0})$ in $\mathbb{C}^{n}$ and $g:\left(\mathbb{C}^{n}, \underline{0}\right) \rightarrow(\mathbb{C}, 0)$ a map with an isolated critical point. Let $I(W)$ be the ideal in $\mathcal{O}_{n, \underline{0}}$ consisting of the germs of functions vanishing on $W$, and let $\Theta_{n, \underline{0}}$ be the $\mathcal{O}_{n, \underline{0}}$-module of germs of holomorphic vector fields on $\left(\mathbb{C}^{n}, \underline{0}\right)$. Denote by $\Theta_{W}$ those vector fields that are tangent to $W$, that is:

$$
\Theta_{W}=\left\{\psi \in \Theta_{n}: \mathrm{d} h=\psi h \in I(W) \forall h \in I(W)\right\} .
$$

Then the Bruce-Roberts Milnor number of $g$ with respect to $W$ is defined as

$$
\mu_{B R}(g, W)=\operatorname{dim}_{\mathbb{C}} \frac{\mathcal{O}_{n}}{\mathrm{~d} g\left(\Theta_{W, \underline{0}}\right)} .
$$

This number is finite if and only if $g$ is $\mathcal{R}_{W}$-finitely determined. Given any point $x \in U$, we denote by $\Theta_{W}(x)$ the linear subspace of $T_{x} U$ generated by the vectors $\delta(x)$ with $\delta \in \Theta_{W, x}$. A complex analytic stratification $\left\{W_{\alpha}\right\}$ of $W$ is called logarithmic if for each $x \in W_{\alpha}$ one has that the tangent space $T_{x} W_{\alpha}$ coincides with $\Theta_{W, x}$. The germ $(W, \underline{0})$ is said to be holonomic if, for some neighborhood $U$ of $\underline{0}$ in $\mathbb{C}^{n}$, there is a logarithmic stratification (with finitely many strata). In this case, by [4, Proposition 1.10], in a sufficiently small neighborhood of 
the origin, this stratification is Whitney regular. In this case, the logarithmic subvariety of $W$ is defined as $L C(W):=\bigcup_{\alpha} \overline{C\left(W_{\alpha}\right)}$, where $C\left(W_{\alpha}\right)$ is the conormal space of $W_{\alpha}$, that is, the subspace of $T^{*} \mathbb{C}^{n}$ given by all forms vanishing on the tangent bundle $T W_{\alpha}$.

Now we consider $g$ and $V$ as before and suppose that $(V, \underline{0})$ is holonomic for the logarithmic stratification $\left\{V_{\alpha}\right\}$ of $(V, \underline{0})$. Consider the induced stratification $\left\{V_{\alpha} \cap F_{t}\right\}$ in $V \cap F_{t}$, which is also logarithmic. If $L C\left(V \cap F_{t}\right)$ is Cohen-Macaulay at $\left(\underline{0}, d\left(g \mid F_{t}\right)(\underline{0})\right)$ then, by [4, Corollary 5.8], we obtain

$$
\mu_{B R}\left(g, F_{t}\right)=\sum_{V_{\alpha} \cap F_{t}} n_{\alpha} c_{\alpha},
$$

where $n_{\alpha}$ is the multiplicity of the component $\overline{C\left(V_{\alpha} \cap F_{t}\right)}$ and $c_{a}$ is the number of critical points of a Morsification of $g$ in $F_{f}$ that are contained in that stratum. Thus we see that the GSV-index of $g$ and the Bruce-Roberts invariant are both defined by the number of critical points of a Morsification of $g$. From the results by N. Grulha in [17] we get also a relation between these invariants and the Euler obstruction of $g$.

6. The classical setting. Consider map-germs $f:=\left(f_{1}, \ldots, f_{k}\right):\left(\mathbb{C}^{n+k}, \underline{0}\right) \rightarrow$ $\left(\mathbb{C}^{k}, 0\right)$ and $g:\left(\mathbb{C}^{n+k}, \underline{0}\right) \rightarrow(\mathbb{C}, 0)$ as before. We assume further that $X$ is non-singular away from $\underline{0}$. The above discussion implies that if $f$ has the Thom $a_{f}$-property and $g$ has an isolated singularity in $X$ and in $V(f)$, then $f$ and $(f, g)$ have associated Milnor-Lê fibrations, and their corresponding Euler characteristics are related by Theorem 3.1. This formula involves the number of critical points of a Morsification of $g$ on the Milnor fibre $F_{f}$. We now say a few words about this latter invariant.

Let $\mathcal{C}(f, g)$ be the union of $\operatorname{Crit}(f)$, the critical points of $f$, and the points in $X \backslash\{\operatorname{Crit}(f)\}$ where the fibers of $f$ are tangent to those of $g$. In other words, using the language of René Thom in [35], away from $\operatorname{Crit}(f)$, we are looking at the contacts of the foliations $\mathcal{F}$ and $\mathcal{G}$ defined by the fibers of $f$ and $g$, respectively.

For instance, if $g$ were a general linear form, the set $\mathcal{C}(f, g)$ would be a classical polar variety.

In general $\mathcal{C}(f, g)$ is the set of critical points of the map $(f, g):\left(\mathbb{C}^{n+k}, \underline{0}\right) \rightarrow\left(\mathbb{C}^{k+1}, 0\right)$, so it is always complex analytic, defined by the determinants of all $(k+1) \times(k+1)$ minors of the Jacobian matrix of $(f, g)$. Clearly one has that the critical points of $g$ in $F_{f}$ are the points in the intersection $\mathcal{C}(f, g) \cap F_{f}$.

Let $x_{o}$ be a point in $\mathcal{C}(f, g) \cap F_{f}$. Notice that $x_{o}$ is a regular point of $f$. Let us choose a local chart $U$ for $X$ around $x$, so that the bundle $T f$ tangent to the fibers of $f$ is holomorphically trivial on $U$, and let $\xi_{1}, \ldots, \xi_{n}$ be holomorphic vector fields in $U$ that span the bundle $T f$. Then $\mathcal{C}(f, g) \cap U$ is defined by the equations $\left\langle\nabla g, \xi_{i}\right\rangle=0, i=1, \ldots, n$, where $\nabla g$ is the gradient of $g$ and $\langle$,$\rangle is the usual Hermitian product. These are 2 n$ real analytic equations, hence generically $\mathcal{C}(f, g)$ has real codimension $2 n$, and it is complex analytic. Thus generically it has complex dimension $k$ and $\mathcal{C}(f, g) \cap F_{f}$ consists of isolated points.

For instance, if $k=1$, then $\mathcal{C}(f, g)$ is a complex curve in $X$ passing through the origin. This curve necessarily has an isolated singularity at $\underline{0}$ and each branch of this local germ meets 
transversally each Milnor fibre $F_{f}$ at a finite number of points. The curve is reduced precisely when the contacts of the foliations $\mathcal{F}$ and $\mathcal{G}$ are all generic, i.e., when the critical points of $\left.g\right|_{F_{f}}$ are all Morse critical points.

Notice that if $x \in \mathcal{C}(f, g) \cap F_{f}$ is an isolated critical point of $\left.g\right|_{F_{f}}$, where the fibre $F_{f}$ is $f^{-1}(t) \cap X \cap \mathbb{B}_{\varepsilon}$ for some regular value $t$, then the local contribution of $x$ for the invariant $c\left(\left.g\right|_{F_{f}}\right)$ is given by the algebraic invariant:

$$
\operatorname{dim}_{\mathbb{C}} \frac{\mathcal{O}_{X, \underline{0}}}{\left(f-t, \operatorname{Jac}_{k+1}(f, g)\right)},
$$

where $\operatorname{Jac}_{k+1}(f, g)$ denotes the ideal generated by the determinants of all the $(k+1)$ minors of the corresponding Jacobian matrix.

Assume now that $X$ is $\mathbb{C}^{n+k}$ and the germs $f$ and $(f, g)$ are both ICIS, i.e., isolated complete intersection singularities.

Let us recall now some algebraic background as developed in [13]. Let $\mathcal{X} \subset \mathbb{C}^{n+k} \times \mathbb{C}^{k}$ be the analytic space defined by $\mathcal{X}:=V\left(f_{1}-t_{1}, \ldots, f_{k}-t_{k}\right)$, where $t_{1}, \ldots, t_{k}$ denote the variables in $\mathbb{C}^{k}$. Let $\pi_{2}: \mathbb{C}^{n+k} \times \mathbb{C}^{k}$ be the projection into the second factor, and denote its restriction to $\mathcal{X}$ by $p$. Since $\mathcal{X}$ is a complete intersection it is Cohen-Macaulay and hence $p$ is flat because all the fibers are equidimensional of pure dimension $n$.

Now consider the free module $\mathcal{O}_{\mathcal{X}}^{k+1}$ of $(k+1)$-tuples of $\mathbb{C}$-valued functions on $\mathcal{X}$. Let $\mathcal{M}:=J M\left(f_{1}, \ldots, f_{k}, g\right) \subseteq \mathcal{O}_{\mathcal{X}}^{k+1}$ be the Jacobian module of $f_{1}, \ldots, f_{k}, g$. That is, $J M\left(f_{1}, \ldots, f_{k}, g\right)$ is the $\mathcal{O}_{\mathcal{X}}$-module generated by the columns of the corresponding Jacobian matrix.

Let $\mathcal{S}:=\operatorname{Supp}\left(\frac{\mathcal{O}_{\mathcal{X}}^{k+1}}{\mathcal{M}}\right)$ be the support of the $\mathcal{O}_{\mathcal{X}}$-module $\frac{\mathcal{O}_{\mathcal{X}}^{k+1}}{\mathcal{M}}$, that is, $\mathcal{S}$ is the subvariety of $\mathcal{X}$ defined by $\operatorname{Jac}_{k+1}(f, g)$. In our case, the induced map

$$
p: \mathcal{S} \rightarrow \mathbb{C}^{k},
$$

is a finite surjective morphism, since by hypothesis $g$ has an isolated critical point at $V(f)$ and $f$ has the Thom property. This implies that the module $\mathcal{M}$ defines a finite colength submodule $\mathcal{M}(\underline{t})$ in $\mathcal{O}_{X(\underline{t})}^{k+1}$, for each $\underline{t} \in \mathbb{C}^{k}$, where $X(\underline{t})$ denotes the fiber of $p: \mathcal{X} \rightarrow \mathbb{C}^{k}$ at $\underline{t}$.

Thus one may define the total Buchsbaum-Rim multiplicity $e(t)$ as:

$$
e(\underline{t})=\sum_{x \in X(\underline{t})} e_{B R}\left(\mathcal{M}(\underline{t}), \mathcal{O}_{X(\underline{t}), x}^{k+1}\right),
$$

where

$$
e_{B R}\left(\mathcal{M}(\underline{t}), \mathcal{O}_{X(\underline{t}, x}^{k+1}\right)=\lim _{d \rightarrow \infty}\left[\frac{(n+k) !}{d^{n+k}} \operatorname{dim}_{\mathbb{C}}\left(\frac{\operatorname{Sym}_{d}\left(\mathcal{O}_{X(\underline{t}), x}^{k+1}\right)}{\operatorname{Rees}_{d}(\mathcal{M}(\underline{t}))}\right)\right]
$$

is the usual Buchsbaum-Rim multiplicity as defined for example in [20].

Since $p: \mathcal{X} \longrightarrow \mathbb{C}^{k}$ is flat, $X(\underline{t})$ is Cohen-Macaulay for each $\underline{t} \in \mathbb{C}^{k}, p: \mathcal{S} \rightarrow \mathbb{C}^{k}$ is surjective, and $\mathcal{M}$ is generated by $n+(k+1)-1$ elements, we have, by [13, Proposition 1.5], that $e(\underline{t})$ is constant. Theorem 2.4 and Corollaries 4.3 and 4.5 by Buchsbaum and Rim in [5] imply: 


$$
e(\underline{t})=\sum_{x \in X(\underline{t})} \operatorname{dim}_{\mathbb{C}} \frac{\mathcal{O}_{\mathbb{C}^{n+k}, x}}{\left(f_{1}-t_{1}, \ldots, f_{k}-t_{k}, \operatorname{Jac}_{k+1}(f, g)\right) \mathcal{O}_{\mathbb{C}^{n+k}, x}}
$$

where $\left(f_{1}-t_{1}, \ldots, f_{k}-t_{k}, \operatorname{Jac}_{k+1}(f, g)\right) \mathcal{O}_{\mathbb{C}^{n+k}, x}$ denotes the ideal in $\mathcal{O}_{\mathbb{C}^{n+k}, x}$ generated by $f_{1}-t_{1}, \ldots, f_{k}-t_{k}, \operatorname{Jac}_{k+1}(f, g)$.

Hence the right hand side of (1) is independent of $\underline{t}$. This, together with Theorem 3.1, says that in this case one has

$$
\chi\left(F_{f}\right)=\chi\left(F_{f, g}\right)+(-1)^{n} \operatorname{dim}_{\mathbb{C}} \frac{\mathcal{O}_{n+k, \underline{0}}}{\left(f, \operatorname{Jac}_{k+1}(f, g)\right)},
$$

or equivalently:

$$
\mu(f)+\mu(f, g)=\operatorname{dim}_{\mathbb{C}} \frac{\mathcal{O}_{n+k, \underline{0}}}{\left(f, \operatorname{Jac}_{k+1}(f, g)\right)},
$$

which is the classical Lê-Greuel formula.

\section{REFERENCES}

[ 1 ] J.-P. BRasselet, D. T. LÊ And J. SEAde, Euler obstruction and indices of vector fields, Topology 39 (2000), 1193-1208.

[2] J.-P. Brasselet, D. Massey, A. J. Parameswaran and J. Seade, Euler obstruction and defects of functions on singular varieties, London Math. Soc. (2) 70 (2004), no. 1, 59-76.

[ 3 ] J.-P. Brasselet, J. Seade And T. Suwa, Vector fields on singular varieties, Lecture Notes in Math. 1987, Springer-Verlag, Berlin, 2009.

[ 4 ] J. W. Bruce And R. M. Roberts, Critical points of functions on analytic varieties, Topology 27 (1988), no. 1, 57-90.

[ 5 ] D. A. Buchsbaum And D. S. Rim, A generalized Koszul complex. II: Depth and multiplicity, Trans. Am. Math. Soc. 111 (1964), 197-224.

[6] J. L. Cisneros-Molina, N. G. Grulha JR. And J. Seade, On the topology of real analytic maps, Internat. J. Math. 25 (2014), no. 7, 1450069, 30 pp.

[ 7 ] J. DAmon, Higher multiplicities and almost free divisors and complete intersections, Mem. Amer. Math. Soc. 123 (1996), no. 589, x+113 pp.

[ 8 ] N. Dutertre And N. G. Grulha JR., Lê-Greuel type formula for the Euler obstruction and applications, Adv. Math. 251 (2014), 127-146.

[9] W. Ebeling And S. Gusein-Zade, On the index of a vector field at an isolated singularity, The Arnoldfest (Toronto, ON, 1997), 141-152, Fields Inst. Commun. 24, A. M. S., Providence, RI, 1999.

[10] V. Ebeling And S. M. Gusein-Zade, Indices of 1-forms on an isolated complete intersection singularity, Moscow Math. J. 3 (2003), no. 2, 439-455.

[11] V. Ebeling And S. M. Gusein-Zade, Radial index and Euler obstruction of a 1-form on a singular variety, Geom. Dedicata 113 (2005), 231-241.

[12] T. GAFFNEY, Non-isolated complete intersection singularities and the $A_{f}$ condition, Singularities I, 85-93, Contemp. Math. 474, A. M. S., Providence, RI, 2008.

[13] T. GafFNEY AND S. Kleiman, Specialization of integral dependence for modules, Invent. Math. 137 (1999), no. 3, 541-574.

[14] X. Gómez-Mont, J. SeAde And A. Verjovsky, The index of a holomorphic flow with an isolated singularity, Math. Ann. 291 (1991), 737-751. 
[15] M. Goresky And R. MacPherson, Stratified Morse theory, Ergebnisse der Mathematik und ihrer Grenzgebiete (3) 14, Berlin Springer-Verlag, 1988.

[16] G. M. GREUEL, Der Gauss-Manin Zusammenhang isolierter Singularitäten von vollständigen Durchschnitten, Dissertation, Göttingen, 1973.

[17] N. G. Grulha JR., The Euler obstruction and Bruce-Roberts' Milnor number, Quarterly J. Maths 60 (2009), no. 3, 291-302.

[18] H. Haмm, Lokale topologische Eigenschaften komplexer Räume, Math. Ann. 191 (1971), 235-252.

[19] H. King And D. Trotman, Poincaré-Hopf theorems on stratified sets, Preprint 1996 (unpublished).

[20] S. Kleiman And A. Thorup, A geometric theory of the Buchsbaum-Rim multiplicity, J. Algebra 167 (1994), no. 1, 168-231.

[21] D. T. LÊ, Calculation of Milnor number of isolated singularity of complete intersection, Funct. Anal. Appl. 8 (1974), 127-131.

[22] D. T. LÊ, Some remarks on relative monodromy, In Real and complex singularities (Proc. Ninth Nordic Summer School/NAVF Sympos. Math., Oslo, 1976), 397-403, Sijthoff and Noordhoff, Alphen aan den Rijn, 1977.

[23] D. T. LÊ, Sur les cycles vanouissants des espaces analytiques (French), C. R. Acad. Sci. Paris Sr. A-B 288 (1979), no. 4, 283-285.

[24] D. T. LÊ AND B. TEISSIER, Variétés polaires locales et classes de Chern de variétés singulères, Ann. of Math. 114 (1981), 457-491.

[25] D. MASSEy, Numerical control over complex analytic singularities, Mem. Amer. Math. Soc. 163 (2003), no. 778 , xii+268 pp.

[26] J. J. Nuño-Ballesteros, B. Oréfice AND J. N. TomaZella, The Bruce-Roberts number of a function on a weighted homogeneous hypersurface, Q. J. Math. 64 (2013), no. 1, 269-280.

[27] J. J. NuÑo-Ballesteros, B. ORÉFICE And J. N. Tomazella, The vanishing Euler characteristic of an isolated determinantal variety, Israel J. Math. 197 (2013), no. 1, 475-495.

[28] J. Milnor, Singular points of complex hypersurfaces, Ann. of Math. Stud. 61, Princeton University Press, Princeton, N.J., 1968.

[29] K. S Aito, Calcul algébrique de la monodromie, In "Singularités à Cargèse" (Rencontre Singularités Géom. Anal. Inst. Études Sci., Cargèse, 1972), 195-211, Astérisque, Nos. 7 et 8, Soc. Math. France, Paris, 1973.

[30] M.-H. Schwartz, Champs radiaux sur une stratification analytique, Travaux en Cours 39, Hermann, Paris, 1991.

[31] J. SEADE, On the topology of isolated singularities in analytic spaces, Progr. Math. 241. Birkhäuser Verlag, Basel, 2006.

[32] J. Seade, M. Tibar and A. Verjovsky, Milnor numbers and Euler obstruction, Bull. Braz. Math. Soc. (N.S.) 36 (2005), no. 2, 275-283.

[33] B. TeISSIER, Cycles évanescents, sections planes et conditions de Whitney (French) Singularités à Cargèse (Rencontre Singularités Géom. Anal., Inst. Études Sci., Cargèse, 1972), 285-362, Astérisque, Nos. 7 et 8 , Soc. Math. France, Paris, 1973.

[34] B. TEISSIER, Variétés polaires. II. Multiplicités polaires, sections planes, et conditions de Whitney, In "Algebraic geometry" (La Rábida, 1981), Lecture Notes in Math. 961, 314-491, Springer-Verlag, Berlin, 1982.

[35] R. Tном, Généralization de la théorie de Morse et variétés feuilletées, Ann. Inst. Fourier (Grenoble) 14 (1964), 173-190.

[36] J.-L. Verdier, Stratifications de Whitney et théorème de Bertini-Sard, Invent. Math. 36 (1976), 295-312. 


$\begin{array}{ll}\text { CENTRO DE CIÊNCIAS EXATAS E DA NATUREZA - UFPB } & \text { INSTITUTO DE BIOCIÊNCIAS } \\ \text { CIDADE UNIVERSITÁRIA S/N, JOÃO PESSOA, PB } & \text { LETRAS E CIÊNCIAS EXATAS - UNESP } \\ \text { BRASIL } & \text { RUA CRISTÓVÃO COLOMBO } \\ & 2265, \text { JD. NAZARETH } \\ \text { E-mail address: roberto@ mat.ufpb.br } & \text { SÃo JOSÉ DO RIO PRETO, SP } \\ & \text { BRASIL } \\ & \text { E-mail address: mmorgado@ibilce.unesp.br } \\ & \\ \text { INSTITUTO DE CIÊNCIAS MATEMÁTICAS E DE } & \text { INSTITUTO DE MATEMÁTICAS } \\ \text { COMPUTAÇÃO - USP } & \text { UNIDAD CUERNAVACA - UNAM } \\ \text { AV. TRABALHADOR SÃO-CARLENSE } & \text { AV. UNIVERSIDAD S/N } \\ \text { 400, SÃO CARLOS, SP } & \text { COL. LOMAS DE CHAMILPA } \\ \text { BRASIL } & \text { CUERNAVACA, MORELOS } \\ \text { E-mail address: mjsaia@icmc.usp.br } & \text { MEXICO } \\ & \text { E-mail address: jseade@im.unam.mx }\end{array}$

